\title{
Oxidation of Aromatic Lithium Thiolates into Sulfinate Salts: An Attractive Entry to Aryl Sulfones Labeled with Carbon-11
}

Claudie Martin, Franck Sandrinelli, Cécile Perrio, * Stéphane Perrio* and Marie-Claire Lasne

Laboratoire de Chimie Moléculaire et Thio-Organique (UMR CNRS 6507), ENSICAEN, Université de Caen-Basse Normandie, 6 Boulevard du Maréchal Juin, F-14050 Caen, France

and

Groupe de Développements Méthodologiques en Tomographie par Emission de Positons, CNRS FRE 2698, CEA DSV UMR 2E, Université de Caen-Basse Normandie, Centre Cyceron, Boulevard Henri Becquerel, F-14074 Caen, France

e-mail: perrio@cyceron.fr or perrio@ensicaen.fr

\section{Supporting Information}

General Conditions

Procedures and Spectral Data of Compounds 6-22 


\section{General Conditions}

\section{Non-Radioactive Chemistry}

THF was freshly distilled under a nitrogen atmosphere from sodium benzophenone ketyl. All other reagents and solvents were used as received from commercial sources. All reactions were performed under a nitrogen atmosphere unless stated otherwise. All glassware and syringes used with thiols (stench) were washed with bleach after use. Alkylations of sulfinate salts in sealed tube were carried out in $15 \mathrm{~mL}$ ace pressure tubes. The concentration of MeLi was determined by titration against diphenylacetic acid. ${ }^{1}$ 2-Phenylsulfonyl-3-phenyloxaziridine 5 was prepared in two steps from benzaldehyde and benzenesulfonamide using a published procedure. ${ }^{2}$ Benzenesulfinic acid was obtained by acidification of sodium benzenesulfinate with $6 \mathrm{~N}$ aqueous $\mathrm{H}_{2} \mathrm{SO}_{4}$ solution. ${ }^{3}$

IR spectra were recorded on a FT-IR instrument. All chemical shifts ( $\delta)$ and coupling constants are quoted in parts per million $(\mathrm{ppm})$ and $\mathrm{Hertz}(\mathrm{Hz})$ respectively. The following abbreviations are used to designate the multiplicity of the signals: $\mathrm{s}=$ singlet; $\mathrm{d}=$ doublet; $\mathrm{t}=$ triplet; $\mathrm{q}=$ quartet; $\mathrm{m}=$ multiplet, and combinations thereof. ${ }^{1} \mathrm{H}$ spectra are referenced either to TMS $\left(0.0, \mathrm{CDCl}_{3}\right)$ or $\mathrm{HOD}\left(4.78, \mathrm{D}_{2} \mathrm{O}\right) .{ }^{13} \mathrm{C}$ chemical shifts are referenced to $\mathrm{CDCl}_{3}(77.00$, $\left.\mathrm{CDCl}_{3}\right)$ or 1,4-dioxane $\left(67.40, \mathrm{D}_{2} \mathrm{O}\right)$. Mass spectra were recorded on a GC/MS/MS instrument. Only peaks of an intensity $>10 \%$ (except decisive ones) are listed. Melting points were obtained

1 Kofron, W. G.; Baclawski, L. M. J. Org. Chem. 1976, 41, 1879-1880.

2 (a) Vishwakarma, L. C.; Stringer, O. D.; Davis, F. A. Org. Synth. 1988, 66, 203-210. (b) Davis F. A.; Chattopadhyay, S.; Towson, J. C.; Lal, S.; Reddy, T. J. Org. Chem. 1988, 53, 2087-2089.

3 Marcantoni, E.; Mecozzi, T.; Petrini, M. J. Org. Chem. 2002, 67, 2989-2994. 
on a capillary apparatus and are uncorrected. Reactions were purified by chromatography column with silica gel Si $60(0.040-0.063 \mathrm{~nm})$. Thin layer chromatography was carried out on silica gel $60 \mathrm{~F}_{254}(1.1 \mathrm{~mm})$ with spot detection under $\mathrm{UV}$ light or through $\mathrm{I}_{2}$ or $\mathrm{KMnO}_{4}$ oxidation.

\section{Radioactive Chemistry}

$\left[{ }^{11} \mathrm{C}\right]$ Carbon dioxide was prepared by the ${ }^{14} \mathrm{~N}(\mathrm{p}, \alpha){ }^{11} \mathrm{C}$ nuclear reaction using nitrogen gas target at Cyceron Center in Caen. ${ }^{11} \mathrm{C}$-Alkyl iodides were prepared according to the described methods. ${ }^{4}$ Radioactivity was measured with a Radioisotope Calibrator. Bombardment was carried out for 1-3 min with a $1.5 \mathrm{~mA}$ beam of $16 \mathrm{MeV}$ protons to give $145-370 \mathrm{MBq}$ (4-10 mCi) of $\left[{ }^{11} \mathrm{C}_{\mathrm{CO}_{2}}\right.$ and $36-180 \mathrm{MBq}(1-5 \mathrm{mCi})$ of $\left[{ }^{11} \mathrm{C}\right] \mathrm{alkyl}$ iodides. Identification of the labeled compounds and determination of the radiochemical purity were carried out by radio TLC using an automatic TLC-linear analyser and authentic stable isotope samples as reference. The plates were developed in petroleum ether/ethyl acetate mixtures (7:3, eluent A or 1:1, eluent B). Preparative HPLC was carried out on a HPLC system consisting of intelligent pump, UV detector, chromato-integrator in series with a scintillation detector. Purifications were performed on a normal-phase column (Nucleosil 100-7, $250 \times 4.6 \mathrm{~mm}$ ) eluted at $5 \mathrm{~mL} \cdot \mathrm{min}^{-1}$ with $n$ heptane/ethyl acetate (7:3, eluent $C$ or 1:1, eluent $D)$ at $\lambda=254 \mathrm{~nm}$. Rf and $t_{R}$ for sulfones 10-15 and sulfinic esters 16-21 are given in Table 1.

4 (a) Crouzel, C.; Långström, B.; Pike, V. W.; Coenen, H. H. Appl. Radiat. Isot. 1987, 38, 601-603. (b) Kawamura, K.; Elsinga, P. H.; Kobayashi, T.; Ishii, S-I.; Wang, W-F.; Matsuno, K.; Vaalburg, W.; Ishiwata, K. Nucl. Med. Biol. 2003, 30, 273-284. (c) Guillouet, S.; Barre, L.; Gourand, F.; Lasne, M. C.; Rault, S. J. Labelled Compd. Radiopharm. 1996, 38, 367-371. 
General Procedure for the Synthesis of Lithium Sulfinates 6-9. To a cooled $\left(-78^{\circ} \mathrm{C}\right)$ solution of the aromatic thiol $(1 \mathrm{mmol})$ in THF $(1.5 \mathrm{~mL})$ was added dropwise MeLi $(0.69 \mathrm{~mL}$ of a 1.6 $\mathrm{N}$ solution in $\left.\mathrm{Et}_{2} \mathrm{O}, 1.1 \mathrm{mmol}\right)$. After stirring the solution at $-78^{\circ} \mathrm{C}$ for $15 \mathrm{~min}$, a solution of $N$-sulfonyloxaziridine $5(548 \mathrm{mg}, 2.1 \mathrm{mmol})$ in THF $(1.2 \mathrm{~mL})$ was added dropwise very slowly (exothermic reaction). The reaction mixture was warmed to $-40{ }^{\circ} \mathrm{C}(15 \mathrm{~min})$ and around this temperature the mixture became cloudy. The solution was then stirred at $-10{ }^{\circ} \mathrm{C}$ (ice $/ \mathrm{NaCl}$ bath) for 15 min and AcOEt $(30 \mathrm{~mL})$ was added. The sulfinate salt was extracted with distilled $\mathrm{H}_{2} \mathrm{O}$ (3 $\times 3 \mathrm{~mL})$. The combined aqueous extracts were washed with AcOEt $(4 \times 30 \mathrm{~mL})$, concentrated, and dried overnight under high vacuum to provide quantitatively the pure sulfinate salt. ${ }^{5}$ Further purification was not required.

Benzenesulfinic acid lithium salt (6). Obtained by oxidation of lithium thiolate generated from thiophenol. White solid. ${ }^{1} \mathrm{H}$ NMR $\left(\mathrm{D}_{2} \mathrm{O}, 250 \mathrm{MHz}\right) \delta: 7.60-7.57(\mathrm{~m}, 2 \mathrm{H}), 7.51-7.41(\mathrm{~m}$, $3 \mathrm{H}) .{ }^{13} \mathrm{C}$ NMR $\left(\mathrm{D}_{2} \mathrm{O}, 62.9 \mathrm{MHz}\right) \delta: 153.7,130.9,129.4,123.7$.

4-Fluorobenzenesulfinic acid lithium salt (7). Obtained by oxidation of lithium thiolate generated from 4-fluorothiophenol. White solid. ${ }^{1} \mathrm{H}$ NMR $\left(\mathrm{D}_{2} \mathrm{O}, 250 \mathrm{MHz}\right) \delta: 7.58-7.43(\mathrm{~m}, 2 \mathrm{H})$, 7.18-7.06 (m, 2H). ${ }^{19} \mathrm{~F}$ NMR $\left(\mathrm{D}_{2} \mathrm{O}, 235.3 \mathrm{MHz}\right) \delta:-111.7(\mathrm{~m}, 1 \mathrm{~F}) .{ }^{13} \mathrm{C}$ NMR $\left(\mathrm{D}_{2} \mathrm{O}, 62.9 \mathrm{MHz}\right) \delta$ : $161.9\left(\mathrm{~d}, J_{\mathrm{CF}}=246.5 \mathrm{~Hz}\right), 147.7\left(\mathrm{~d}, J_{\mathrm{CF}}=2.5 \mathrm{~Hz}\right), 124.0\left(\mathrm{~d}, J_{\mathrm{CF}}=8.8 \mathrm{~Hz}\right), 114.1\left(\mathrm{~d}, J_{\mathrm{CF}}=22.6\right.$ $\mathrm{Hz})$.

2-(Methoxycarbonyl)benzenesulfinic acid lithium salt (8). Obtained by oxidation of lithium thiolate generated from thiosalicylic acid methyl ester. White solid. ${ }^{1} \mathrm{H}$ NMR $\left(\mathrm{D}_{2} \mathrm{O}, 250\right.$ MHz) $\delta: 7.84(\mathrm{dd}, J=7.6$ and $1.2 \mathrm{~Hz}, 1 \mathrm{H}), 7.72(\mathrm{dd}, J=7.6$ and $1.2 \mathrm{~Hz}, 1 \mathrm{H}), 7.62(\mathrm{dt}, J=7.6$

The weight of the sulfinate salt was slightly superior because of remaining water. 
and $1.2 \mathrm{~Hz}, 1 \mathrm{H}), 7.41(\mathrm{dt}, J=7.6$ and $1.2 \mathrm{~Hz}, 1 \mathrm{H}), 3.85(\mathrm{~s}, 3 \mathrm{H}) .{ }^{13} \mathrm{C} \mathrm{NMR}\left(\mathrm{D}_{2} \mathrm{O}, 62.9 \mathrm{MHz}\right) \delta$ : $169.5,155.0,133.7,130.5,130.3,128.2,122.9,53.1$.

2-Pyridinesulfinic acid lithium salt (9). Obtained by oxidation of lithium thiolate generated from 2-mercaptopyridine. White solid. ${ }^{1} \mathrm{H}$ NMR $\left(\mathrm{D}_{2} \mathrm{O}, 250 \mathrm{MHz}\right) \delta: 8.38$ (ddd, $J=4.9$, $1.7 \mathrm{~Hz}$ and $0.9 \mathrm{~Hz}, 1 \mathrm{H}), 7.89(\mathrm{dt}, J=7.55$ and $1.7 \mathrm{~Hz}, 1 \mathrm{H}), 7.67(\mathrm{dt}, J=7.55$ and $1.2 \mathrm{~Hz}, 1 \mathrm{H})$, 7.36 (ddd, $J=7.55,4.9$ and $1.2 \mathrm{~Hz}, 1 \mathrm{H}) .{ }^{13} \mathrm{C}$ NMR $\left(\mathrm{D}_{2} \mathrm{O}, 62.9 \mathrm{MHz}\right) \delta: 170.3,148.8,138.7$, $125.9,117.4$

\section{General Procedures for Lithium Sulfinate Alkylation in Non-radioactive Chemistry}

Method A: In a round-bottomed flask fitted with a condenser and a magnetic stirrer, a mixture of the sulfinate salt 6-9 $(1 \mathrm{mmol})$, tetrabutylammonium bromide $(25 \mathrm{mg})$ and the appropriate alkyl halide $(1.5 \mathrm{mmol}, 1.5 \mathrm{eq})$ was heated to $80-85^{\circ} \mathrm{C}$ for $24 \mathrm{~h}$ in a solvent system consisting of toluene $(0.3 \mathrm{~mL})$, acetone $(0.3 \mathrm{~mL})$ and water $(0.4 \mathrm{~mL})$. After being cooled to room temperature, the reaction mixture was poured into a saturated aqueous $\mathrm{NaCl}$ solution $(2 \mathrm{~mL})$ and the product was extracted with $\mathrm{Et}_{2} \mathrm{O}(3 \times 30 \mathrm{~mL})$. The combined organic extracts were dried over $\mathrm{MgSO}_{4}$, filtered and concentrated. The resulting crude product was then analysed by ${ }^{1} \mathrm{H}$ NMR. When methyl iodide or benzyl bromide was employed as the electrophile, the sulfone arising from $S$-alkylation of the lithium sulfinate was detected as the sole product. In contrast, contamination with the sulfinic ester $(<5 \%)$ was observed using ethyl iodide. The product was then subjected to column chromatography to afford the pure sulfone (and the pure ester if formed).

Method B: In a sealed tube, a solution of the sulfinate salt 6-9 (1 mmol) and the appropriate alkyl halide (1.5 mmol, $1.5 \mathrm{eq})$ in a 4:1 mixture of THF and water ( $3 \mathrm{~mL}$ ) was heated with an oil at $120^{\circ} \mathrm{C}$ for $5 \mathrm{~min}$. The work-up is then similar to that described above in Method A. 
1-Methylsulfonylbenzene (10). ${ }^{6}$ Obtained by alkylation of sulfinate 6 with methyl iodide according to Method A. Purified by column chromatography on silica gel (petroleum ether/ethyl acetate, 9:1, $\mathrm{Rf}=0.10$ ). Yield: $75 \%$. White solid. Mp: $87{ }^{\circ} \mathrm{C}$ (diethyl ether) (Litt. ${ }^{6} \mathrm{Mp}: 85-87$ $\left.{ }^{\circ} \mathrm{C}\right) .{ }^{1} \mathrm{H}$ NMR $\left(\mathrm{CDCl}_{3}, 250 \mathrm{MHz}\right) \delta: 7.97-7.94(\mathrm{~m}, 2 \mathrm{H}), 7.70-7.55(\mathrm{~m}, 3 \mathrm{H}), 3.06(\mathrm{~s}, 3 \mathrm{H}) .{ }^{13} \mathrm{C}$ NMR $\left(\mathrm{CDCl}_{3}, 62.9 \mathrm{MHz}\right) \delta: 140.6,133.7,129.3,127.3,44.5 . \mathrm{IR}\left(\mathrm{KBr}, \mathrm{cm}^{-1}\right):$ v: 3090, 3022, 2926, 1448, 1408, 1328, 1086. MS (EI) m/z 156 (M+ 46$), 141$ (38), 77 (100), 51 (22).

1-Benzylsulfonylbenzene (11). ${ }^{6}$ Obtained by alkylation of sulfinate 6 with benzyl bromide according to Method A. In this particular case, ethyl acetate was used as the extraction solvent instead of diethyl ether. Purified by column chromatography on silica gel (petroleum ether/ethyl acetate, 9:1, $\mathrm{Rf}=0.30$ ). Yield: $85 \%$. White solid. Mp: $146{ }^{\circ} \mathrm{C}$ (diethyl ether) $\left[\mathrm{Litt}^{7} \mathrm{Mp}: 146{ }^{\circ} \mathrm{C}\right.$ (ethyl alcohol)]. ${ }^{1} \mathrm{H}$ NMR $\left(\mathrm{CDCl}_{3}, 250 \mathrm{MHz}\right) \delta: 7.64-7.57(\mathrm{~m}, 3 \mathrm{H}), 7.47-7.41(\mathrm{~m}, 2 \mathrm{H})$, 7.35-7.21 (m, 3H), 7.09-7.05 (m, 2H), $4.30(\mathrm{~s}, 2 \mathrm{H}) .{ }^{13} \mathrm{C} \mathrm{NMR}\left(\mathrm{CDCl}_{3}, 62.9 \mathrm{MHz}\right) \delta: 137.9$, 133.7, 130.8, 128.8, 128.7, 128.6, 128.5, 128.1, 62.9. IR $\left(\mathrm{KBr}, \mathrm{cm}^{-1}\right): v: 3064,3030,2968,2918$, 1448, 1304, 1290, 1152, 1126, 1082. MS (EI) m/z $232\left(\mathrm{M}^{+}, 11\right), 91$ (100), 77 (14).

1-Methylsulfonyl-4-fluorobenzene (12). ${ }^{8}$ Obtained by alkylation of sulfinate 7 with methyl iodide according to Method B. Purified by column chromatography on silica gel (petroleum ether/ethyl acetate, $\mathrm{Rf}=0.28)$. Yield: $70 \%$. White solid. $\mathrm{Mp}: 80^{\circ} \mathrm{C} .{ }^{1} \mathrm{H}$ NMR $\left(\mathrm{CDCl}_{3}\right.$, $250 \mathrm{MHz}) \delta: 7.9-8.00(\mathrm{~m}, 2 \mathrm{H}), 7.23-7.29(\mathrm{~m}, 2 \mathrm{H}), 3.06(\mathrm{~s}, 3 \mathrm{H}) .{ }^{19} \mathrm{~F}$ NMR $\left(\mathrm{CDCl}_{3}, 235.3 \mathrm{MHz}\right)$ $\delta:-103,9\left(\mathrm{tt}, J_{\mathrm{HF}}=7.5\right.$ and $\left.5 \mathrm{~Hz}, 1 \mathrm{~F}\right) .{ }^{13} \mathrm{C} \mathrm{NMR}\left(\mathrm{CDCl}_{3}, 62.9 \mathrm{MHz}\right) \delta: 165.7\left(\mathrm{~d}, J_{\mathrm{CF}}=257 \mathrm{~Hz}\right)$,

\footnotetext{
$6 \quad$ Shaabani, A.; Mirzaei, P.; Naderi, S.; Lee, D. G. Tetrahedron 2004, 60, 11415-11420.

$7 \quad$ Shirota, Y.; Nagai, T.; Tokura, N. Tetrahedron 1967, 23, 639-648.

8 Mieloszynski, J. L.; Weber, J. V.; Schneider, M.; Paquer, D.; Born, M.; Parc, G. Sulfur Lett. 1988, 8, 27-30.
} 
$136.6\left(\mathrm{~d}, J_{\mathrm{CF}}=3.1 \mathrm{~Hz}\right), 130.2\left(\mathrm{~d}, J_{\mathrm{CF}}=9.4 \mathrm{~Hz}\right), 116.6\left(\mathrm{~d}, J_{\mathrm{CF}}=23 \mathrm{~Hz}\right), 44.6 . \mathrm{IR}\left(\mathrm{NaCl}, \mathrm{cm}^{-1}\right): v$ : 2930, 1590, 1540, 1490, 1406, 1312. MS (EI) m/z $174\left(\mathrm{M}^{+}, 22\right), 159$ (41), 111 (28), 95 (100).

1-Ethylsulfonyl-4-fluorobenzene (13). ${ }^{9}$ Obtained as the major product by alkylation of sulfinate 7 with ethyl iodide according to Method A. Purified by column chromatography on silica gel (petroleum ether/ethyl acetate, 4:1, $\mathrm{Rf}=0.24$ ). Yield: $76 \%$. Colorless oil. ${ }^{1} \mathrm{H}$ NMR $\left(\mathrm{CDCl}_{3}, 250 \mathrm{MHz}\right) \delta: 7.97-7.85(\mathrm{~m}, 2 \mathrm{H}), 7.31-7.22(\mathrm{~m}, 2 \mathrm{H}), 3.13(\mathrm{q}, J=7.4 \mathrm{~Hz}, 2 \mathrm{H}), 1.28(\mathrm{t}, J=$ $7.4 \mathrm{~Hz}, 3 \mathrm{H}) .{ }^{19} \mathrm{~F} \mathrm{NMR}\left(\mathrm{CDCl}_{3}, 235.3 \mathrm{MHz}\right) \delta:-104.0\left(\mathrm{tt}, J_{\mathrm{HF}}=8.0\right.$ and $\left.5.0 \mathrm{~Hz}, 1 \mathrm{~F}\right) .{ }^{13} \mathrm{C} \mathrm{NMR}$ $\left(\mathrm{CDCl}_{3}, 62.9 \mathrm{MHz}\right) \delta: 166.1\left(\mathrm{~d}, J_{\mathrm{CF}}=256.6 \mathrm{~Hz}\right), 134.4\left(\mathrm{~d}, J_{\mathrm{CF}}=3.1 \mathrm{~Hz}\right), 131.0\left(\mathrm{~d}, J_{\mathrm{CF}}=9.4 \mathrm{~Hz}\right)$, $116.5\left(\mathrm{~d}, J_{\mathrm{CF}}=22.0 \mathrm{~Hz}\right), 50.7,7.4 . \mathrm{IR}\left(\mathrm{NaCl}, \mathrm{cm}^{-1}\right): v: 3106,3074,2984,2942,1592,1496$, 1456, 1318, 1146, 1088. MS (EI) m/z $188\left(\mathrm{M}^{+}, 41\right), 159$ (44), 95 (84), 91 (100), 77 (30), 75 (36), $65(22)$.

2-(Methylsulfonyl)benzoic acid methyl ester (14). ${ }^{10}$ Obtained by alkylation of sulfinate 8 with methyl iodide according to Method A. Purified by column chromatography on silica gel (petroleum ether/ethyl acetate, $6: 4, \mathrm{Rf}=0.28$ ). Yield: $77 \%$. White solid. Mp: $64{ }^{\circ} \mathrm{C}$ (petroleum ether) [Litt. ${ }^{10} \mathrm{Mp}: 60-61{ }^{\circ} \mathrm{C}$ (chloroform/petroleum ether)]. ${ }^{1} \mathrm{H}$ NMR $\left(\mathrm{CDCl}_{3}, 250 \mathrm{MHz}\right) \delta$ : 8.16-8.12 (m, 1H), 7.72-7.65 (m, 3H), $3.98(\mathrm{~s}, 3 \mathrm{H}), 3.35(\mathrm{~s}, 3 \mathrm{H}) .{ }^{13} \mathrm{C}$ NMR $\left(\mathrm{CDCl}_{3}, 62.9 \mathrm{MHz}\right)$ $\delta: 167.5,139.2,133.5,133.1,131.3,129.9,129.7,53.2,44.9 . \mathrm{IR}\left(\mathrm{KBr}, \mathrm{cm}^{-1}\right): v: 3034,3010$, 2954, 2932, $1732(\mathrm{C}=\mathrm{O}), 1434,1294,1152,1060 . \mathrm{MS}(\mathrm{EI}) \mathrm{m} / \mathrm{z} 214\left(\mathrm{M}^{+}, 16\right), 199$ (16), 183 (100), 135 (8), 91 (18), 77 (42), 51 (13).

9 Mignot, A.-A.; Rumpf, P. Bull. Soc. Chim. Fr. 1968, 435-436.

$10 \quad$ Bowden, K.; Rehman, S. J. Chem. Res. (S) 1997, 406-407. 
2-(Methylsulfonyl)pyridine (15). ${ }^{11}$ Obtained by alkylation of sulfinate 9 with methyl iodide according to Method A. Purified by column chromatography on silica gel (petroleum ether/ethyl acetate, 6:4, $\mathrm{Rf}=0.3)$. Yield: $66 \%$. Brown oil. ${ }^{1} \mathrm{H} \mathrm{NMR}\left(\mathrm{CDCl}_{3}, 250 \mathrm{MHz}\right) \delta: 8.75$ (dd, $J=4.9$ and $1.7 \mathrm{~Hz}, 1 \mathrm{H}), 8.10(\mathrm{dd}, J=7.9$ and $0.9 \mathrm{~Hz}, 1 \mathrm{H}), 7.98(\mathrm{dt}, J=7.9$ and $1.7 \mathrm{~Hz}, 1 \mathrm{H})$, $7.56(\mathrm{ddd}, J=7.9,4.9$ and $0.9 \mathrm{~Hz}, 1 \mathrm{H}), 3.24(\mathrm{~s}, 3 \mathrm{H}) .{ }^{13} \mathrm{C} \mathrm{NMR}\left(\mathrm{CDCl}_{3}, 62.9 \mathrm{MHz}\right) \delta: 158.1$, 150.1, 138.3, 127.4, 121.1, 40.0. IR $\left(\mathrm{NaCl}, \mathrm{cm}^{-1}\right):$ v: 3056, 3012, 2930, 1580, 1456, 1428, 1306 , 1166, 1084. MS (EI) $m / z 157\left(\mathrm{M}^{+}, 1\right), 93$ (38), 78 (100), 51 (50).

Benzenesulfinic acid methyl ester (16). ${ }^{12}$ Obtained by treatment of diphenyl disulfide with bromine in the presence of methanol as described in the literature. ${ }^{12}$ Purified by distillation under reduced pression. $\mathrm{Eb}=90-95{ }^{\circ} \mathrm{C}(4 \mathrm{mmHg})\left[\mathrm{Litt} .{ }^{12} \mathrm{~Eb}=79-83{ }^{\circ} \mathrm{C}(0.3 \mathrm{mmHg})\right]$. Yield: $81 \%$. Colorless oil. ${ }^{1} \mathrm{H}$ NMR $\left(\mathrm{CDCl}_{3}, 250 \mathrm{MHz}\right) \delta$ : 7.73-7.69 (m, 2H,), 7.56-7.52 (m, 3H), 3.47 (s, 3H). ${ }^{13} \mathrm{C} \mathrm{NMR}\left(\mathrm{CDCl}_{3}, 62.9 \mathrm{MHz}\right) \delta: 143.7,132.0,129.0,125.2,49.4 . \mathrm{IR}\left(\mathrm{NaCl}, \mathrm{cm}^{-1}\right): v: 3060$, 1582, 1540, 1478, 1128. MS (EI) m/z $156\left(\mathrm{M}^{+}, 58\right), 141$ (10), 125 (51), 77 (100).

Benzenesulfinic acid benzyl ester (17). ${ }^{13}$ Obtained by reaction of benzenesulfinic acid with thionyl chloride supported on silica gel and benzylic alcohol in solid phase conditions. ${ }^{14}$ Purified by column chromatography on silica gel (petroleum ether/ethyl acetate, 70:30, $\mathrm{Rf}=0.7$ ). Yield: $18 \%$. Colorless oil. ${ }^{1} \mathrm{H}$ NMR $\left(\mathrm{CDCl}_{3}, 250 \mathrm{MHz}\right) \delta: 7.78-7.58(\mathrm{~m}, 2 \mathrm{H}),, 7.56-7.52(\mathrm{~m}, 3 \mathrm{H})$,

11 Baudin, J. B.; Hareau, G.; Julia, S. A.; Lorne, R.; Ruel, O. Bull. Soc. Chim. Fr 1993, 130, $856-878$.

12 Resek, J. E.; Meyers, A. I. Tetrahedron Lett. 1995, 36, 7051-7054.

13 Harpp, D. N.; Friedlander, B. T.; Larsen, C.; Steliou, K.; Stockton, A. J. Org. Chem. 1978, 43, 3481-3485.

14 Hajipour, A. R.; Mallakpour, S. E.; Afrousheh, A. Phosphorus, Sulfur \& Silicon 2000, $160,67-75$. 
7.34-7.26 (m, 5H), 5.05 and $4.57\left(2 \mathrm{~d}, J=11.3 \mathrm{~Hz}, 1 \mathrm{H}\right.$ each) ${ }^{13} \mathrm{C}$ NMR $\left(\mathrm{CDCl}_{3}, 62.9 \mathrm{MHz}\right) \delta$ 144.2, 135.1, 131.9, 128.7, 128.2, 128.1, 125.0, 65.6. IR $\left(\mathrm{NaCl}, \mathrm{cm}^{-1}\right): v: 3062,1498,1456,1444$, 1132, 1080. MS (CI, acetonitrile) $\mathrm{m} / z 233\left(\mathrm{MH}^{+}, 100\right), 125$ (27), 91 (51), 77 (62).

4-Fluorobenzenesulfinic acid methyl ester (18). The protocol was adapted from a general one reported in the literature. ${ }^{15}$ A solution of 4-fluorothiophenol $(385 \mathrm{mg}, 3 \mathrm{mmol})$ and [bis(trifluoroacetoxy)iodo]benzene $(2.58 \mathrm{~g}, 2 \mathrm{eq}, 6 \mathrm{mmol})$ in methanol $(75 \mathrm{~mL})$ was heated under reflux for $4 \mathrm{~h}$. After cooling to room temperature, saturated brine was added $(75 \mathrm{~mL})$ and the reaction mixture was extracted diethyl ether $(3 \times 25 \mathrm{~mL})$. The organic extracts were combined, washed with saturated brine $(2 \times 25 \mathrm{~mL})$, dried over $\mathrm{MgSO}_{4}$, filtered and concentrated. Subsequent separation of the desired sulfinate and liberated iodobenzene by column chromatography on silica gel (petroleum ether/ethyl acetate, $7: 1, \mathrm{Rf}=0.89$ for iodobenzene, $\mathrm{Rf}=$ 0.35 for the sulfinate) allowed the isolation of pure methyl sulfinate $\mathbf{1 8}(359 \mathrm{mg}, 2.06 \mathrm{mmol}$, 69\%) as a colorless oil. ${ }^{1} \mathrm{H}$ NMR $\left(\mathrm{CDCl}_{3}, 250 \mathrm{MHz}\right) \delta$ : 7.74-7.69 (m, 2H), 7.26-7.20 (m, 2H), 3.49 (s, 3H). ${ }^{19} \mathrm{~F} \mathrm{NMR}\left(\mathrm{CDCl}_{3}, 235.3 \mathrm{MHz}\right) \delta:-107.08$ (tt, $J_{\mathrm{HF}}=9$ and $\left.5 \mathrm{~Hz}, 1 \mathrm{~F}\right) .{ }^{13} \mathrm{C} \mathrm{NMR}$ $\left(\mathrm{CDCl}_{3}, 62.9 \mathrm{MHz}\right) \delta: 165.2\left(\mathrm{~d}, J_{\mathrm{CF}}=253 \mathrm{~Hz}\right), 140.00\left(\mathrm{~d}, J_{\mathrm{CF}}=3 \mathrm{~Hz}\right), 128.1\left(\mathrm{~d}, J_{\mathrm{CF}}=9 \mathrm{~Hz}\right)$, $116.53\left(\mathrm{~d}, J_{\mathrm{CF}}=22 \mathrm{~Hz}\right), 49.75$. MS (EI) $\mathrm{m} / z 174\left(\mathrm{M}^{+}, 55\right), 143(100), 115$ (18), 95 (29), 84 (29). HRMS $m / z$ calcd for $\mathrm{C}_{7} \mathrm{H}_{7} \mathrm{FO}_{2} \mathrm{~S} 174.0151$, found 174.0148 .

4-Fluorobenzenesulfinic acid ethyl ester (19). ${ }^{16}$ Obtained as the minor product by alkylation of sulfinate $\mathbf{6}$ with ethyl iodide according to Method A. Purified by column chromatography on silica gel (petroleum ether/ethyl acetate, $4: 1, \mathrm{Rf}=0.68$ ). Yield: $3 \%$. Colorless oil. ${ }^{1} \mathrm{H}$ NMR ( $\left.\mathrm{CDCl}_{3}, 250 \mathrm{MHz}\right) \delta: 7.75-7.69(\mathrm{~m}, 2 \mathrm{H}), 7.26-7.19(\mathrm{~m}, 2 \mathrm{H}), 3.74$ (dq, $J=10.0$ and

15 Xia, M.; Chen, Z.-C. Synth. Commun. 1997, 27, 1321-1326.

16 Evans, B. J.; Doi, J. T.; Musker, W. K. J. Org. Chem. 1990, 55, 2337-2344. 
$7.1 \mathrm{~Hz}, 1 \mathrm{H}), 4.11(\mathrm{dq}, J=10.0$ and $7.1 \mathrm{~Hz}, 1 \mathrm{H}), 1.29(\mathrm{t}, J=7.1 \mathrm{~Hz}, 3 \mathrm{H}) .{ }^{19} \mathrm{~F}$ NMR $\left(\mathrm{CDCl}_{3}, 235.3\right.$ $\mathrm{MHz}) \delta:-107.4\left(\mathrm{tt}, J_{\mathrm{HF}}=8.0\right.$ and $\left.5.0 \mathrm{~Hz}, 1 \mathrm{~F}\right) .{ }^{13} \mathrm{C} \mathrm{NMR}\left(\mathrm{CDCl}_{3}, 62.9 \mathrm{MHz}\right) \delta: 176.7\left(\mathrm{~d}, J_{\mathrm{HF}}=\right.$ $252.8 \mathrm{~Hz}), 152.4\left(\mathrm{~d}, J_{\mathrm{CF}}=3.2 \mathrm{~Hz}\right), 128.1\left(\mathrm{~d}, J_{\mathrm{CF}}=9.4 \mathrm{~Hz}\right), 116.9\left(\mathrm{~d}, J_{\mathrm{CF}}=22.6 \mathrm{~Hz}\right), 61.5,15.9 . \mathrm{IR}$ $\left(\mathrm{NaCl}, \mathrm{cm}^{-1}\right): v: 3000,2962,1492,1260,1094 . \mathrm{MS}(\mathrm{EI}) \mathrm{m} / \mathrm{z} 188\left(\mathrm{M}^{+}, 26\right), 160(35), 143(65), 96$ (100), 75 (33).

2-[(Methoxymethyl)sulfanyl]benzoic acid methyl ester $(\mathbf{2 2}) \cdot{ }^{17}$ To a cooled $\left(-78{ }^{\circ} \mathrm{C}\right)$ solution of thiosalicylic acid methyl ester $(673 \mathrm{mg}, 4 \mathrm{mmol})$ in THF (6 mL), MeLi (5.65 mL of a $0.78 \mathrm{~N}$ solution in $\mathrm{Et}_{2} \mathrm{O}, 1.1 \mathrm{mmol}$ ) was added dropwise. The reaction mixture was stirred at -78 ${ }^{\circ} \mathrm{C}$ for $15 \mathrm{~min}$ and then treated with iodomethyl methyl ether (1.03 g, $\left.6 \mathrm{mmol}, 1.5 \mathrm{eq}\right)$ dissolved in THF (4.8 mL). The reaction mixture was warmed slowly to room temperature over $4 \mathrm{~h}$ and then treated with saturated aqueous $\mathrm{NH}_{4} \mathrm{Cl}$ solution $(30 \mathrm{~mL})$. The product was extracted with diethyl ether $(3 \times 20 \mathrm{~mL})$ and the combined organic extracts were washed with brine $(3 \times 10$ $\mathrm{mL}$ ), dried over $\mathrm{MgSO}_{4}$, filtered and then concentrated to dryness. Purification of the resulting crude product by column chromatography on silica gel, eluting with a 4:1 petroleum ether/ethyl acetate system, afforded the anticipated thioether $22\left(840 \mathrm{mg}, 4 \mathrm{mmol}\right.$, quantitative yield). ${ }^{1} \mathrm{H}$ $\operatorname{NMR}\left(\mathrm{CDCl}_{3}, 250 \mathrm{MHz}\right) \delta: 7.93(\mathrm{dd}, J=7.8$ and $1.5 \mathrm{~Hz}, 1 \mathrm{H}), 7.75(\mathrm{~d}, J=7.8 \mathrm{~Hz}, 1 \mathrm{H}), 7.45(\mathrm{dt}$, $J=7.8$ and $1.5 \mathrm{~Hz}, 1 \mathrm{H}), 7.20(\mathrm{t}, J=7.8 \mathrm{~Hz}, 1 \mathrm{H}), 4.98(\mathrm{~s}, 2 \mathrm{H}), 3.91(\mathrm{~s}, 3 \mathrm{H}), 3.46(\mathrm{~s}, 3 \mathrm{H}) .{ }^{13} \mathrm{C}$ NMR $\left(\mathrm{CDCl}_{3}, 62.9 \mathrm{MHz}\right) \delta: 167.1,140.8,132.7,131.0,128.6,127.7,125.0,75.8,56.3,52.3 . \mathrm{IR}$ $\left(\mathrm{KBr}, \mathrm{cm}^{-1}\right): v: 3010,2938,1710(\mathrm{C}=\mathrm{O}), 1444,1050,1254 . \mathrm{MS}(\mathrm{EI}) \mathrm{m} / \mathrm{z} 212\left(\mathrm{M}^{+}, 100\right), 181(20)$, 136 (31), 108 (35), 105 (21), 69 (31), 45 (69).

17 Kim, D.-W.; Ko, Y. K.; Kim, S. H. Synthesis 1992, 1203-1204. 
2-(Methoxysulfinyl)benzoic acid methyl ester $(\mathbf{2 0}) \cdot{ }^{18}$ Obtained by oxidation of the methoxymethylsulfide 22 prepared above with NBS (2.1 eq) in methanol as reported by Kim. ${ }^{18}$ Purified by column chromatography on silica gel (petroleum ether/ethyl acetate, $4: 1, \mathrm{Rf}=0.68$ ). Yield: $30 \%$. Colorless oil. ${ }^{1} \mathrm{H}$ NMR $\left(\mathrm{CDCl}_{3}, 250 \mathrm{MHz}\right) \delta: 8.23(\mathrm{dd}, J=7.9$ and $0.9 \mathrm{~Hz}, 1 \mathrm{H}), 8.10$ (dd, $J=7.7$ and $1.1 \mathrm{~Hz}, 1 \mathrm{H}), 7.78(\mathrm{dt}, J=7.4$ and $1.2 \mathrm{~Hz}, 1 \mathrm{H}), 7.63(\mathrm{dt}, J=7.6$ and $1.2 \mathrm{~Hz}, 1 \mathrm{H})$, $3.97(\mathrm{~s}, 3 \mathrm{H}), 3.64(\mathrm{~s}, 3 \mathrm{H}) .{ }^{13} \mathrm{C} \mathrm{NMR}\left(\mathrm{CDCl}_{3}, 62.9 \mathrm{MHz}\right) \delta: 165.8,146.4,133.0,131.9,131.3$, 128.5, 125.2, 52.9, 52.7. IR $\left(\mathrm{NaCl}, \mathrm{cm}^{-1}\right): v: 3070,2956,1722(\mathrm{C}=\mathrm{O}), 1438,1292,1018$. MS (EI) $m / z 214\left(\mathrm{M}^{+}, 100\right), 199(31), 185$ (21), 152 (17), 41 (12).

2-Pyridinesulfinic acid methyl ester (21). ${ }^{19}$. Obtained by reaction of 2-mercaptopyridine with NBS (2 eq) in a 1:1 mixture of methanol and dichloromethane according to a literature methodology. ${ }^{19}$ Purified by column chromatography on silica gel (petroleum ether/ethyl acetate, 3:2, $\mathrm{Rf}=0.41)$. Yield: $23 \%$. Colorless oil. ${ }^{1} \mathrm{H} \mathrm{NMR}\left(\mathrm{CDCl}_{3}, 250 \mathrm{MHz}\right) \delta: 8.69(\mathrm{ddd}, J=4.7,1.6$ and $1 \mathrm{~Hz}, 1 \mathrm{H}), 8.0-8.98(\mathrm{~m}, 2 \mathrm{H}), 7.51(\mathrm{ddd}, J=6.9,4.7$ and $2.4 \mathrm{~Hz}, 1 \mathrm{H}), 3.6(\mathrm{~s}, 3 \mathrm{H}) .{ }^{13} \mathrm{C}$ NMR $\left(\mathrm{CDCl}_{3}, 62.9 \mathrm{MHz}\right) \delta: 162.9,150.2,137.8,126.3,120.2,51.2 . \mathrm{IR}\left(\mathrm{NaCl}, \mathrm{cm}^{-1}\right): v: 3050,1576$, 1452, 1424, 1132, 1078, 1040. MS (EI) m/z $158\left(\mathrm{MH}^{+}, 23\right), 127$ (73), 108 (28), 78 (100), 51 (56).

\section{General Procedure for the Synthesis of ${ }^{11} \mathrm{C}$-alkylarylsulfones $\left[{ }^{11} \mathrm{C}\right] 10-15 .{ }^{11} \mathrm{C}$-Alkyl} iodide was trapped at $0{ }^{\circ} \mathrm{C}$ in a $\mathrm{THF} / \mathrm{H}_{2} \mathrm{O}(4: 1)$ solution (total volume $500 \mu \mathrm{L}$ ) containing the sulfinate 6-9 $(5 \mathrm{mg})$. The reactor was closed and the reaction mixture was heated to $150{ }^{\circ} \mathrm{C}$ for 8 min. After cooling at $0{ }^{\circ} \mathrm{C}$ for $1 \mathrm{~min}$, the radioactivity was counted and the volatile compounds were evaporated under a flow of $\mathrm{N}_{2}$. The residue was diluted in a petroleum ether/ethyl acetate

19 Trost, B. M.; Parquette, J. R. J. Org. Chem. 1993, 58, 1579-1581. 
(7:3) mixture $(500 \mu \mathrm{L})$ and the radioactivity was measured again. The crude mixture was filtrated through $\mathrm{MgSO}_{4}$, analyzed by radioTLC and injected onto semipreparative LC. The collected fraction was analyzed by radioTLC and the radioactivity was counted to assess identity and radiochemical purity. Due to the low amounts of radioactivity used, no measurement of specific radioactivity was attempted.

1-[ $\left[{ }^{11} \mathbf{C}\right]-$ Methylsulfonylbenzene $\left(\left[{ }^{11} \mathbf{C}\right] \mathbf{1 0}\right)$. From lithium sulfinate 6 and $\left[{ }^{11} \mathrm{C}^{-} \mathrm{CH}_{3} \mathrm{I}(177\right.$ $\mathrm{MBq}, 4.8 \mathrm{mCi})$, the target compound $\left[{ }^{11} \mathrm{C}\right] \mathbf{1 0}(48 \mathrm{MBq}, 1.3 \mathrm{mCi})$ was isolated in $76 \%$ yield (decay corrected, 31 min total synthesis time including HPLC, radiochemical purity $>99 \%$.).

1- $\left[{ }^{11} \mathrm{C}\right]$-Benzylsulfonylbenzene $\left(\left[{ }^{11} \mathbf{C}\right] \mathbf{1 1}\right)$. From lithium sulfinate 6 and $\left[{ }^{11} \mathrm{C}\right]$ benzyl bromide (44 MBq, $1.2 \mathrm{mCi})$, the target compound $\left[{ }^{11} \mathrm{C}\right] \mathbf{1 1}(7 \mathrm{MBq}, 0.2 \mathrm{mCi})$ was isolated in $62 \%$ yield (decay corrected, 39 min total synthesis time including HPLC, radiochemical purity > $99 \%$.).

1-[ $\left[{ }^{11} \mathrm{C}\right]-$ Methylsulfonyl-4-fluorobenzene $\left(\left[{ }^{11} \mathrm{C}\right] 12\right)$. From lithium sulfinate 7 and $\left[{ }^{11} \mathrm{C}\right] \mathrm{CH}_{3} \mathrm{I}$ (166 MBq, $4.5 \mathrm{mCi})$, the target compound $\left[{ }^{11} \mathrm{C}\right] \mathbf{1 2}(35 \mathrm{MBq}, 0.95 \mathrm{mCi})$ was isolated in $65 \%$ yield (decay corrected, 33 min total synthesis time including HPLC, radiochemical purity $>99 \%$.).

1-[ $\left[{ }^{11} \mathrm{C}\right]$-Ethylsulfonyl-4-fluorobenzene $\left(\left[{ }^{11} \mathbf{C}\right] 13\right)$. From lithium sulfinate 7 and $\left[{ }^{11} \mathrm{C}\right]$ ethyl iodide (82 MBq, $2.2 \mathrm{mCi}$ ), the target compound [ $\left.{ }^{11} \mathrm{C}\right] \mathbf{1 3}(12 \mathrm{MBq}, 0.34 \mathrm{mCi})$ was isolated in $59 \%$ yield (decay corrected, 40 min total synthesis time including HPLC, radiochemical purity > 99\%.).

2-([ $\left[{ }^{11} \mathrm{C}\right]$-Methylsulfonyl)benzoic acid methyl ester $\left(\left[{ }^{11} \mathrm{C}\right] 14\right)$. From lithium sulfinate 8 and $\left[{ }^{11} \mathrm{C}^{-} \mathrm{CH}_{3} \mathrm{I}(181 \mathrm{MBq}, 4.9 \mathrm{mCi})\right.$, the target compound $\left[{ }^{11} \mathrm{C}\right] \mathbf{1 4}(40 \mathrm{MBq}, 1.1 \mathrm{mCi})$ was isolated in $62 \%$ yield (decay corrected, 31 min total synthesis time including HPLC, radiochemical purity $>99 \%$.). 
S13

2-([ $\left[{ }^{11} \mathrm{C}\right]$-Methylsulfonyl)pyridine $\left(\left[{ }^{11} \mathbf{C}\right] 15\right)$. From lithium sulfinate 9 and $\left[{ }^{11} \mathrm{C}\right] \mathrm{CH}_{3} \mathrm{I}(175$ $\mathrm{MBq}, 4.7 \mathrm{mCi})$, the target compound $\left[{ }^{11} \mathrm{C}\right] \mathbf{1 5}(42 \mathrm{MBq}, 1.1 \mathrm{mCi})$ was isolated in $66 \%$ yield (decay corrected, 30 min total synthesis time including HPLC, radiochemical purity $>99 \%$.). 
Table 1. TLC and HPLC data of Compounds 10-21

\begin{tabular}{|c|c|c|c|c|c|c|c|}
\hline \multicolumn{3}{|c|}{ TLC analyses } & \multicolumn{3}{c|}{ HPLC analyses } \\
(eluent: petroleum ether/AcOEt 70:30) & \multicolumn{2}{|c|}{ (eluent: $n$-heptane/AcOEt 70:30) } \\
\hline Sulfones & Rf & Sulfinic & Rf & Sulfones & $t_{R}($ min) & Sulfinic & $t_{R}(\mathrm{~min})$ \\
\hline $\mathbf{1 0}$ & 0.20 & $\mathbf{1 6}$ & 0.48 & $\mathbf{1 0}$ & 10.5 & $\mathbf{1 6}$ & 5.1 \\
$\mathbf{1 1}$ & 0.19 & $\mathbf{1 7}$ & 0.46 & $\mathbf{1 1}$ & 9.6 & $\mathbf{1 7}$ & 4.96 \\
$\mathbf{1 3}$ & 0.17 & $\mathbf{1 8}$ & 0.41 & $\mathbf{1 2}$ & 10.9 & $\mathbf{1 8}$ & 6.3 \\
$\mathbf{1 4}$ & 0.08 & $\mathbf{1 9}$ & 0.24 & $\mathbf{1 3}$ & 29.0 & $\mathbf{1 9}$ & 9.3 \\
$\mathbf{1 5}$ & 0.25 & $\mathbf{2 0}$ & 0.51 & $\mathbf{1 4}$ & 7.6 & $\mathbf{2 0}$ & 4.6 \\
\hline
\end{tabular}



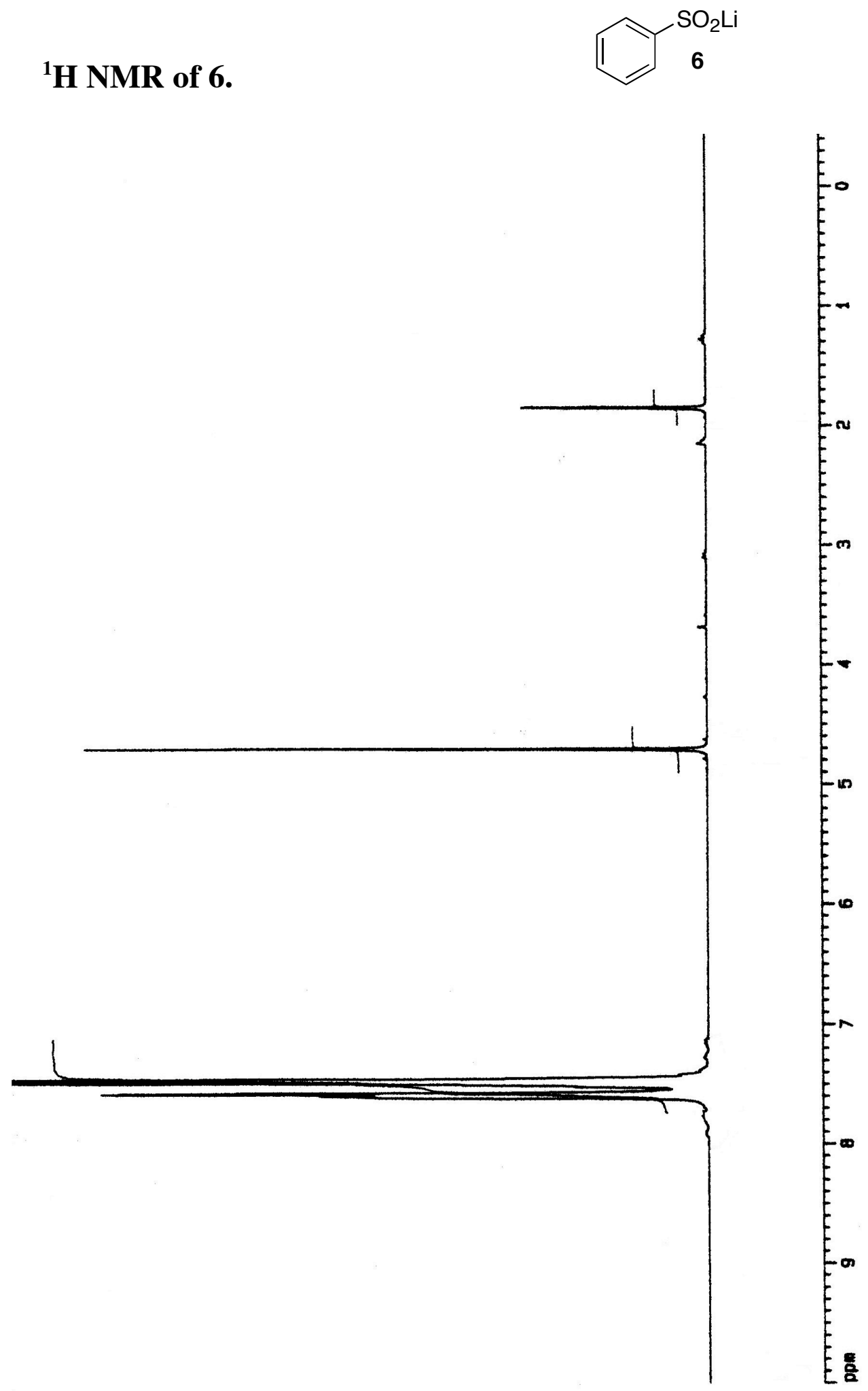
${ }^{13}$ C NMR of 6.

${ }_{6}^{\mathrm{SO}_{2} \mathrm{Li}}$

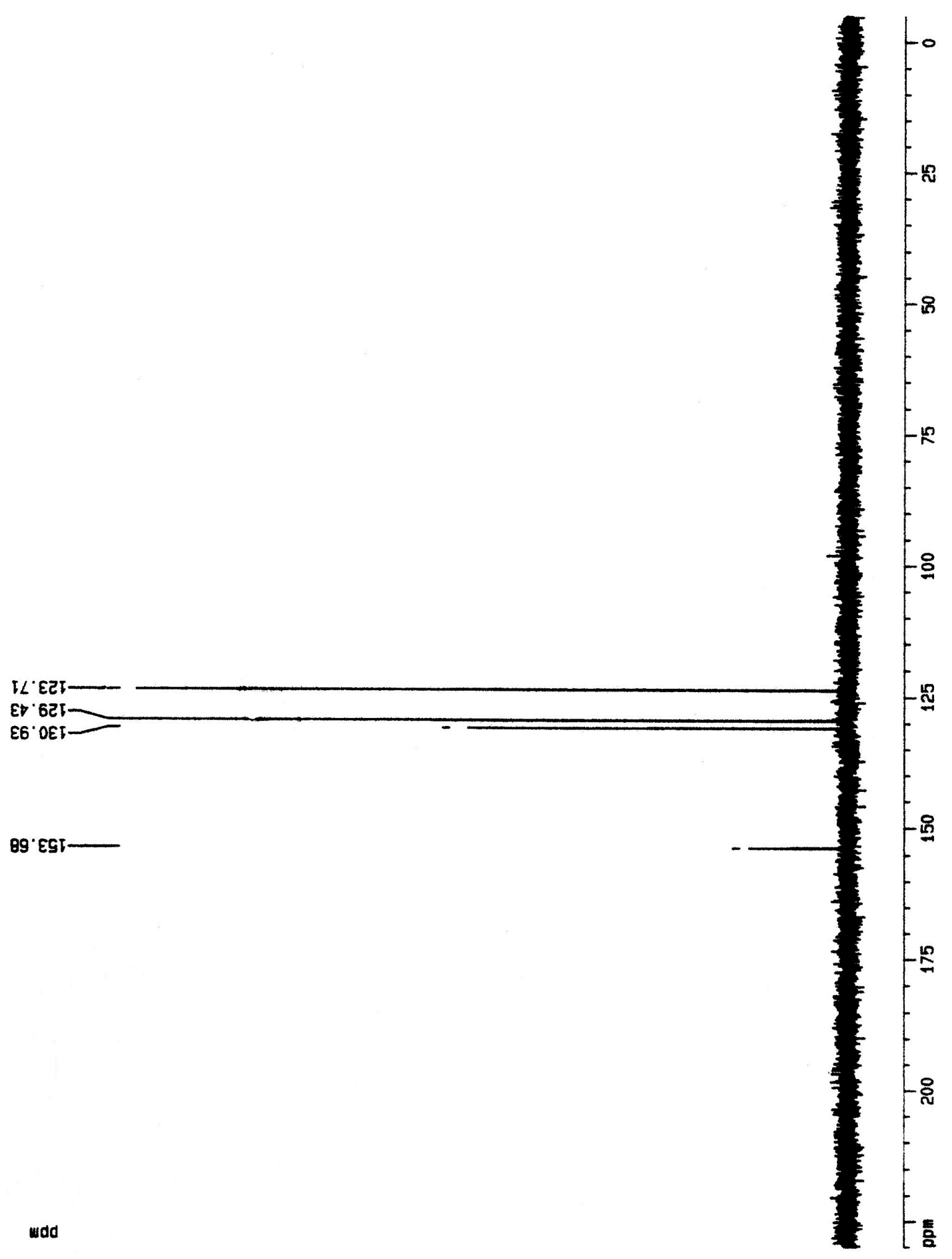


${ }^{1} \mathrm{H}$ NMR of 7.
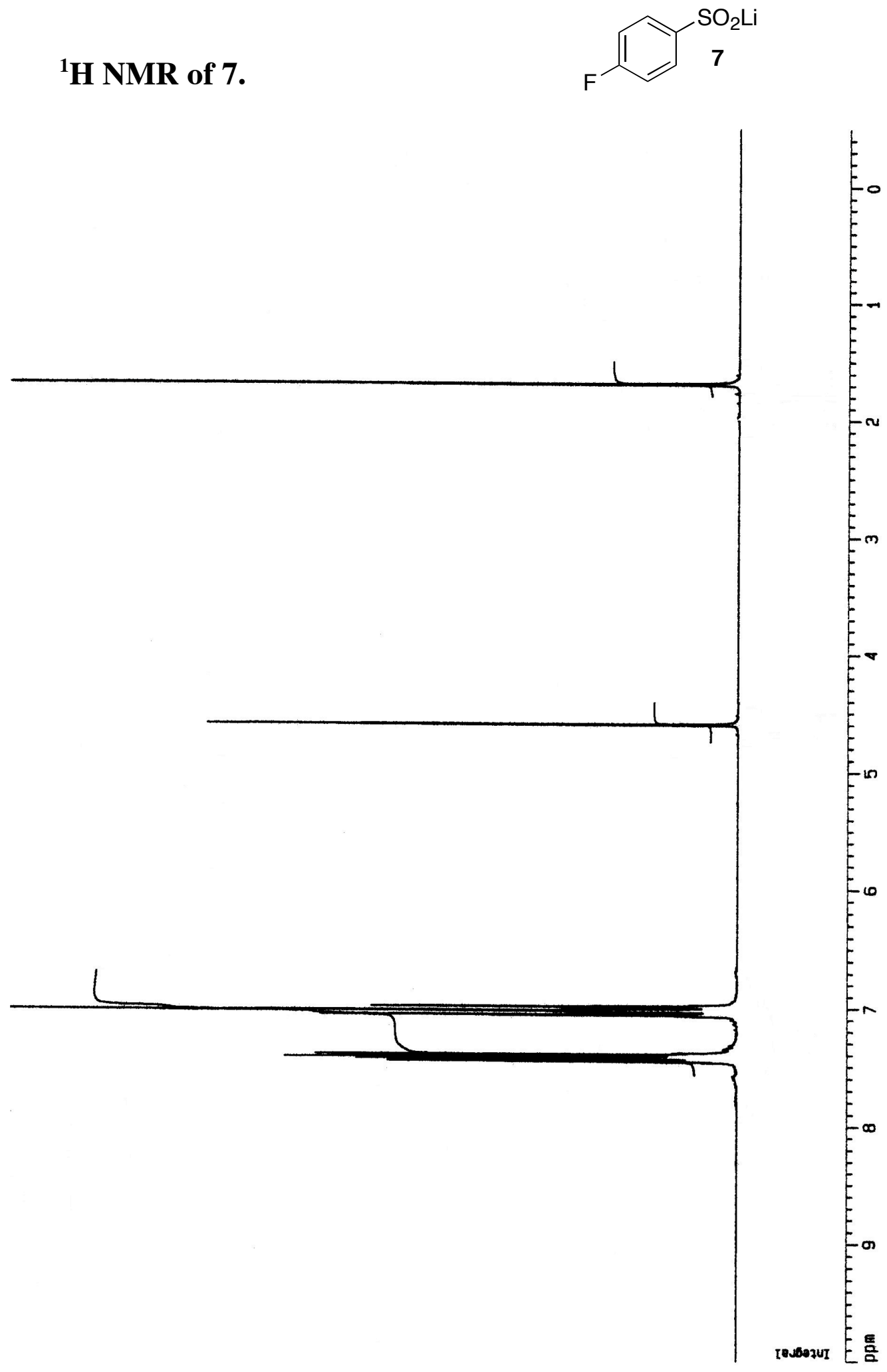
${ }^{13} \mathrm{C}$ NMR of 7.<smiles>O=S(=O)(O[Na])c1ccc(F)cc1</smiles>

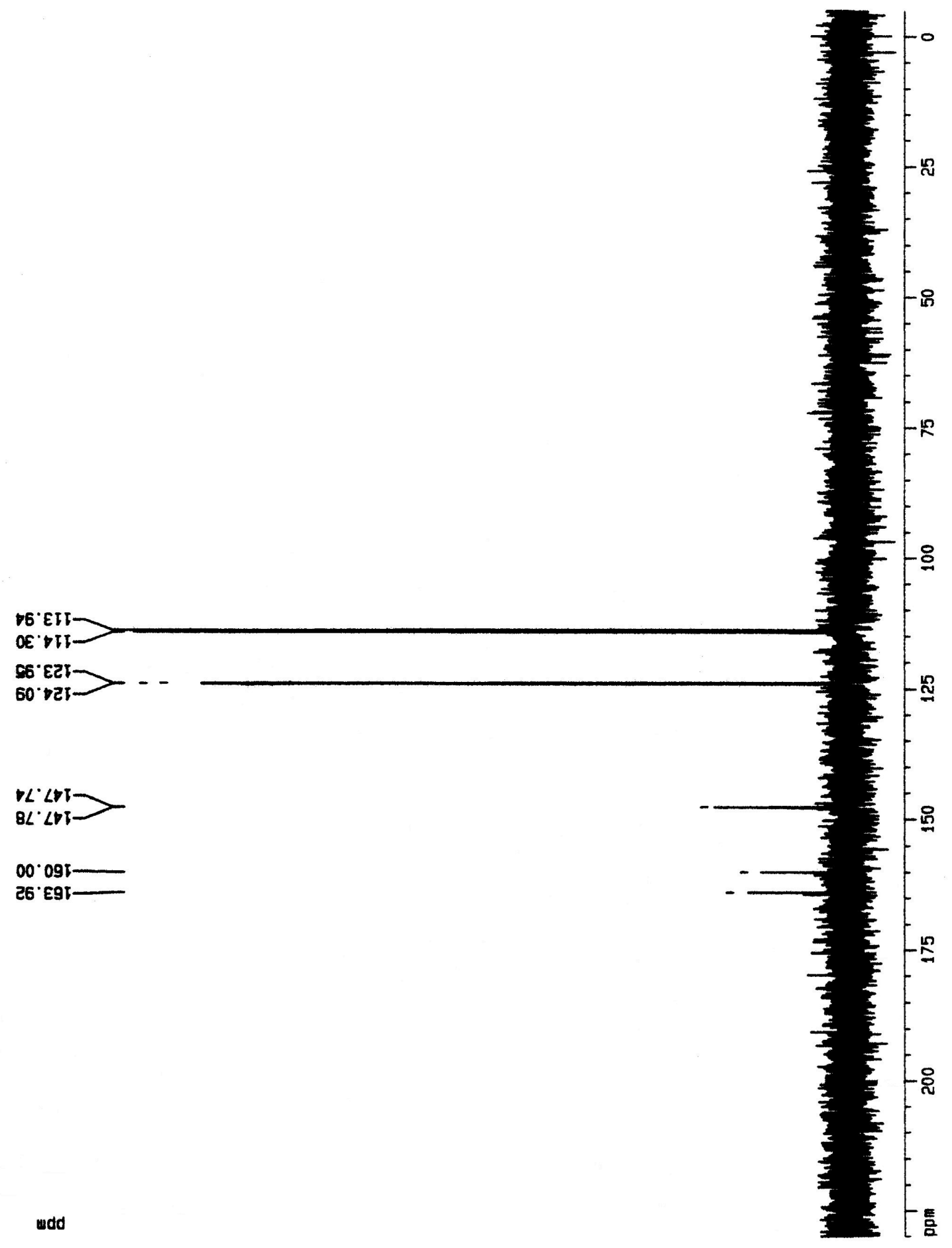


${ }^{1} \mathrm{H}$ NMR of 8.
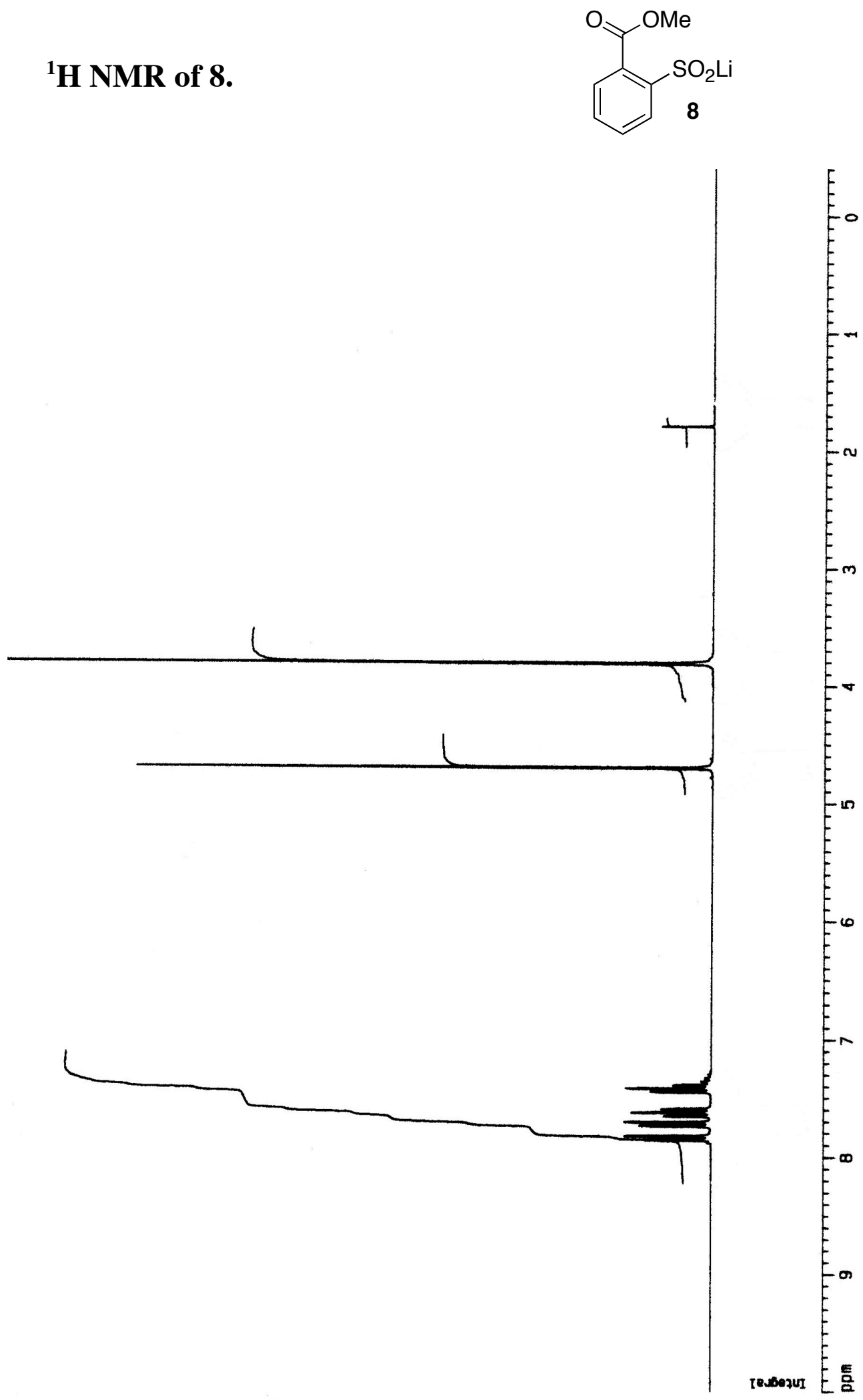
${ }^{13} \mathrm{C}$ NMR of 8 .<smiles>COC(=O)c1ccccc1S(=O)(=O)OC</smiles>

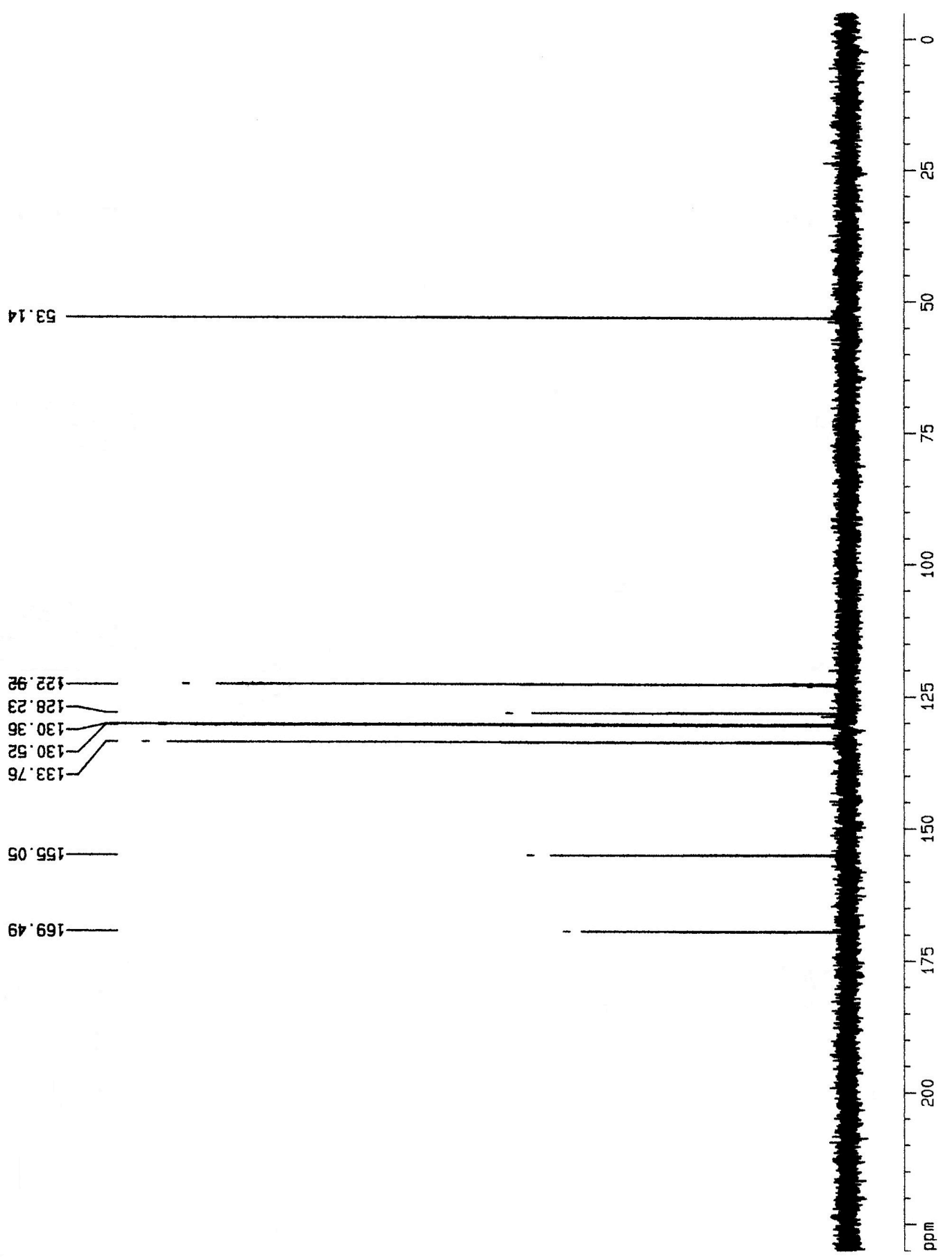


${ }^{1} \mathrm{H}$ NMR of 9.

$\overbrace{=\mathrm{N}}^{\mathrm{SO}_{2} \mathrm{Li}} 9$

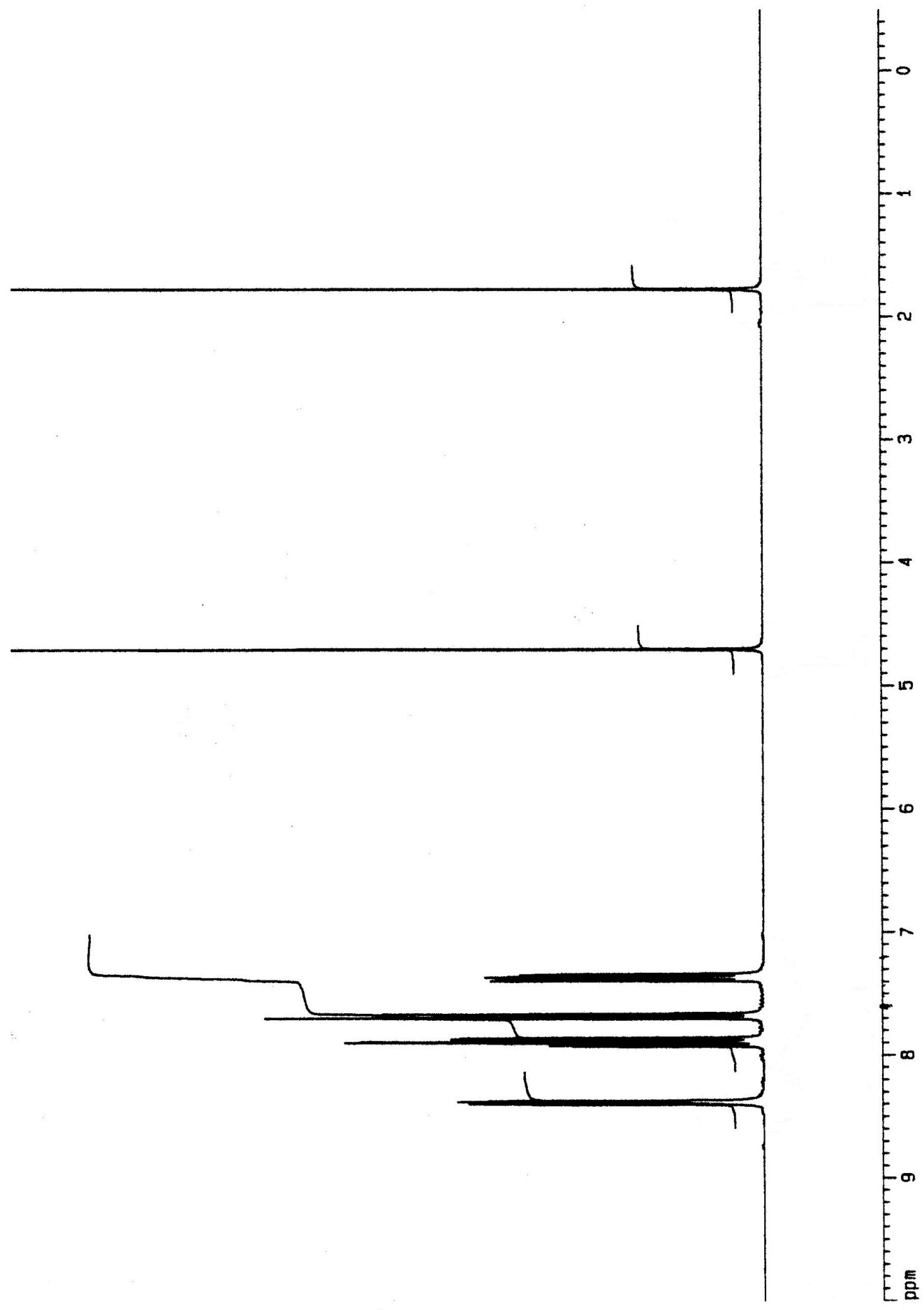


${ }^{13}$ C NMR of 9.<smiles>O=[SH](=O)c1ccccn1</smiles>

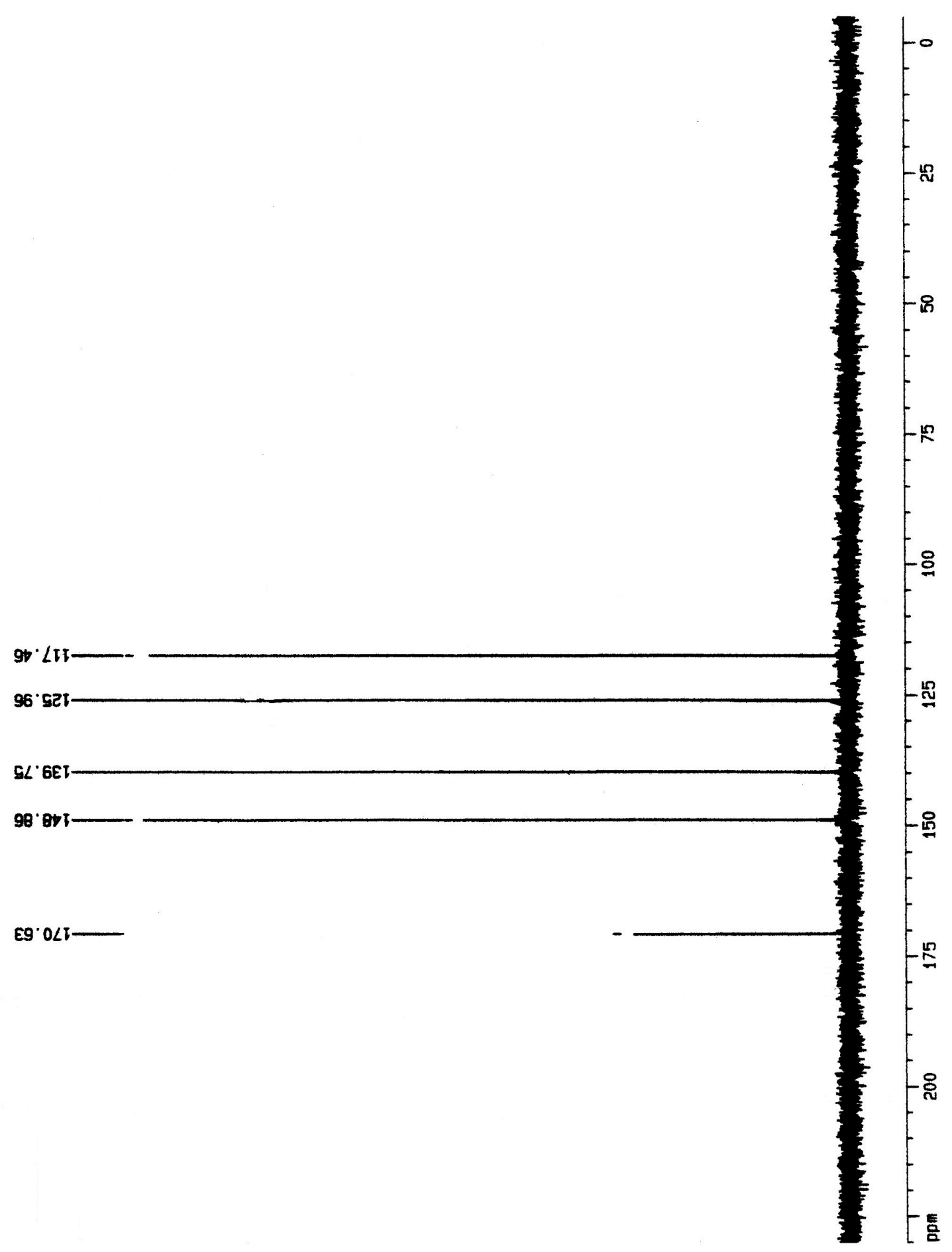

